\title{
Una comparativa de diferentes parámetros en la construcción evolutiva de descriptores para la clasificación de imágenes de texturas de piezas arqueológicas
}

\author{
Julio Antonio Jiménez Falcón ${ }^{1}$, Manuel Ornelas-Rodríguez ${ }^{1}$, \\ Marco Aurelio Sotelo-Figueroa ${ }^{2}$, Pedro Antonio López García ${ }^{3}$, \\ Alfonso Rojas Domínguez ${ }^{1}$, Héctor José Puga Soberanes ${ }^{1}$, \\ Velino Esteves-Jiménez ${ }^{2}$ \\ 1 Tecnológico Nacional de México - Instituto Tecnológico de León, León, Gto., \\ México \\ 2 DCEA-Universidad Guanajuato, Departamento de Estudios Organizacionales, \\ Guanajuato, Gto., México \\ 3 Escuela Nacional de Antropología e Historia, Ciudad de México, México \\ \{julio.jimenez, manuel.ornelas\}@itleon.edu.mx
}

\begin{abstract}
Resumen. En el presente trabajo se muestran los resultados de los descriptores construidos mediante una metodología basada en Gramática Evolutiva. Se hace la comparación de dos parámetros de la propuesta, uno es el uso de los canales de color y la escala de grises en el proceso de construcción de los descriptores, lo cual se llevó a cabo utilizando dos gramáticas tipo Backus-Naur en el proceso evolutivo. La primera gramática usa los canales de color rojo, azul, verde y la escala de grises, mientras que la segunda gramática solo usa la escala de grises. El otro parámetro es la forma de representar los vectores de características de las imágenes procesadas con los descriptores construidos, comparando dos alternativas: el histograma y los estadísticos del histograma. Los descriptores construidos fueron aplicados a la clasificación de imágenes de texturas de piezas arqueológicas procedentes de la cultura egipcia. Se muestran los resultados obtenidos, los cuales fueron analizados estadísticamente.
\end{abstract}

Palabras clave: clasificación de imágenes, construcción de descriptores, gramática evolutiva.

\section{A Comparison between Different Parameters in the Evolutionary Construction of Descriptors for the Classification of Textures Images of Archaeological Pieces}

Abstract. This work shows results about descriptors constructed by a methodology based in Grammatical Evolution. A comparison of two 
parameters of this methodology was made. The first parameter is the use of the color and gray scale channels in the construction process of image descriptors. This was done through a comparison of two Backus-Naur grammars used in the evolutionary process, the first grammar used the red, blue, green and grayscale channels, and the second grammar only used the gray scale. The other parameter was the form to represent the feature vectors of the processed images, two alternatives was compared: the histogram and the histogram statistics. The descriptors constructed were applied to the classification of textures images of archaeological pieces from Egyptian culture. The results obtained are shown, which were analyzed statistically.

Keywords: image classification, feature construction, grammatical evolution.

\section{Introducción}

El análisis y clasificación de texturas representan importantes tareas en el área de visión por computadora. Para obtener resultados eficientes en el proceso de clasificación, es necesario tener descriptores que proporcionen un conjunto de características que representen adecuadamente a la base de datos de imágenes a tratar [1]. Comúnmente esta representación es hecha a mano por expertos en visión por computadora, $[2,3,4,5,6]$, sin embargo, cuando no existe información a priori de la base de datos, la selección de los descriptores adecuados representa un problema. Para solucionar esta dificultad, se han propuesto diferentes alternativas basadas en algoritmos evolutivos, las cuales trabajan sobre la construcción o selección de descriptores de características $[7,8,9,10]$.

En este trabajo, se presenta un método evolutivo para la construcción de descriptores de texturas usando Gramáticas Evolutivas (GE), a partir de la combinación de filtros pasa-baja, pasa-altas y direccionales, y operadores aritméticos. GE requiere una gramática formal, un motor de búsqueda y un proceso de mapeo. También se llevó a cabo una comparativa de dos parámetros, uno es el uso de los canales de color y escala de grises en el proceso de construcción del descriptor contra el uso de únicamente la escala de grises. Otro parámetro es la forma de representar los vectores de características en el proceso de la GE, donde se hizo la comparación entre el histograma y los estadísticos del histograma.

El trabajo está organizado de la siguiente manera: la sección 2 muestra un acercamiento a la GE; la sección 3 describe la metodología que se llevó a cabo; los resultados de la experimentación son reportados en la sección 4 y, por último, la sección 5 presenta las conclusiones. 
Una comparativa de diferentes parámetros en la construcción evolutiva de descriptores...

\section{Gramática evolutiva}

La Gramática Evolutiva (GE) [11] está basada en el conocimiento de la Biología Molecular, como la Programación Genética (PG) [12], pero GE usa una gramática formal relacionada con el problema, un motor de búsqueda que se encarga del proceso evolutivo, y un algoritmo que realiza el proceso de mapeo [13]. GE usa una gramática libre de contexto tipo Backus-Naur (BNF) como gramática formal, la cual es expresada por la tupla $\{\mathrm{N}, \mathrm{T}, \mathrm{P}, \mathrm{S}\}$, donde $\mathrm{N}$ representa los símbolos no terminales, $\mathrm{T}$ son los símbolos terminales, $\mathrm{P}$ es el conjunto de reglas de producción y $\mathrm{S}$ es el símbolo de inicio.

El algoritmo Depth-First (DF) fue usado como proceso de mapeo. Un ejemplo de este proceso es mostrado en la figura 1. DF comienza con el símbolo de inicio y enseguida procede a sustituir el símbolo no terminal ubicado más a la izquierda con un regla de producción seleccionada por la ecuación (1),

$$
\text { Regla }=c \% r,
$$

donde c es un codon del genotipo, $\mathrm{r}$ es el número de reglas de producción válidas para el actual no terminal y $\%$ es el operador módulo. Este proceso continua hasta que el fenotipo generado no contenga más símbolos no terminales.

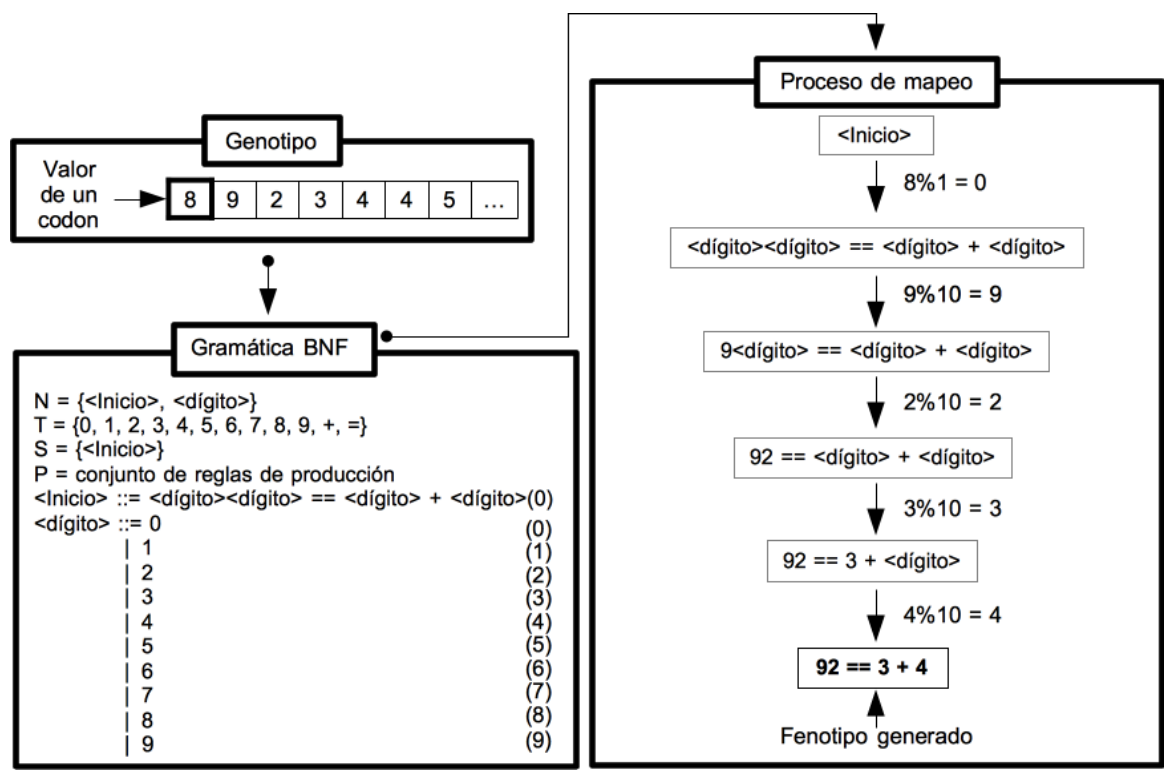

Fig. 1. Un ejemplo del proceso de mapeo de genotipo a fenotipo usando una gramática BNF y el algoritmo DF.

Para este ejemplo, el primer codon es $\mathrm{c}=8$ y el símbolo inicial es $<$ Inicio $>$, el cual tiene un número de regla de producción $r=1$. Aplicando la ecuación 1 
se tiene $8 \% 1=0$, lo que conduce a seleccionar la regla de producción número 0 , dada por $<$ dígito $><$ dígito $>==<$ dígito $>+<$ dígito $>$. Luego, el siguiente codon es $\mathrm{c}=9$ y el símbolo no terminal más a la izquierda es $<$ dígito $>$ con $\mathrm{r}=$ 10 reglas de producción válidas. De la ecuación 1 se obtiene $9 \% 10=9$, lo que nos lleva a sustituir el símbolo no terminal < dígito $>$ por la regla de producción 9, que consiste en el símbolo terminal 9. El proceso de mapeo continua hasta que todos los símbolos no terminales han sido sustituidos por símbolos terminales.

Una vez que la población de genotipos es mapeada a su forma fenotípica, cada solución es evaluada por medio de la función objetivo. Estos valores de rendimiento son usados para evolucionar la población a través de un motor de búsqueda.

\section{Metodología}

Los experimentos realizados en esta investigación se basaron en el modelo de GE usado en [14], el cual es mostrado en la figura 2. En este trabajo, los módulos del modelo consisten en lo siguiente:

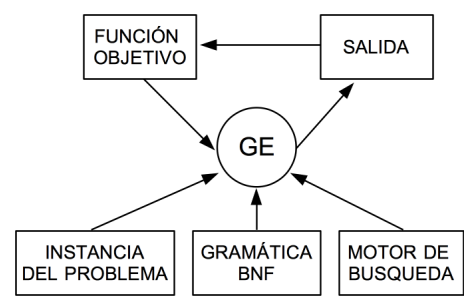

Fig. 2. Diagrama del modelo de GE utilizado en este trabajo [14].

Instancia del problema. Se usó la base de datos utilizada en el trabajo [15], la cual está formada por imágenes digitales de texturas de piezas arqueológicas de la cultura egipcia. Esta base de datos está dividida en dos clases: Arcilla del Nilo con 22 imágenes y Arcilla Marl con 47 imágenes. Cada imagen tiene una resolución original de 3900 x 2900 píxeles, sin embargo, para reducir el costo computacional se redimensionaron a 128 x 128 píxeles mediante interpolación bilineal. La figura 3 muestra ejemplos de las imágenes utilizadas en este experimento.

Gramática BNF. La gramática base utilizada en este trabajo es mostrada en la tabla 1, y sus símbolos terminales son descritos en la tabla 2. Dos variantes de la gramática base fueron usadas (BNF1 y BNF2), donde la diferencia entre ellas es el número de símbolos terminales validos para el símbolo no terminal $<\mathrm{I}>$. BNF1 contiene los canales rojo, verde, azul y la escala de grises (los símbolos terminales Ir, Ig, Ib e Igray), y BNF2 usa únicamente la escala de grises. El trabajo [7] fue usado como base para el diseño de los símbolos terminales. 
Una comparativa de diferentes parámetros en la construcción evolutiva de descriptores...
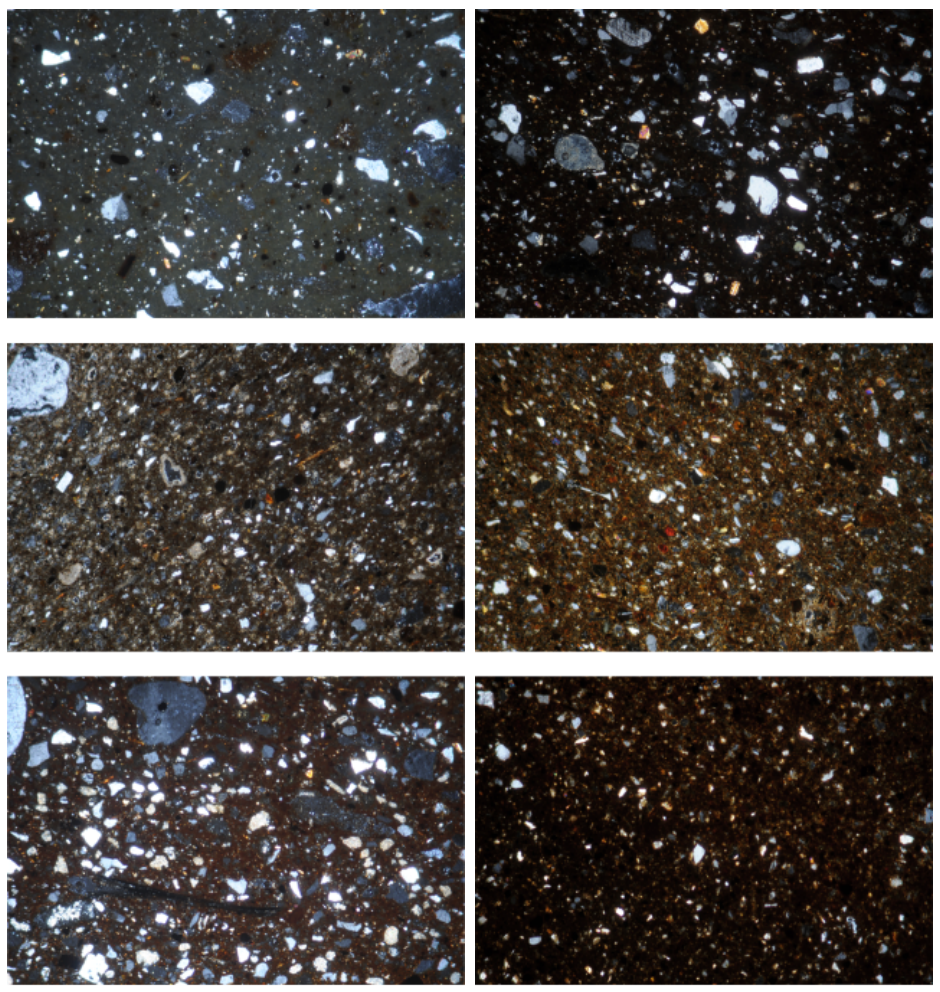

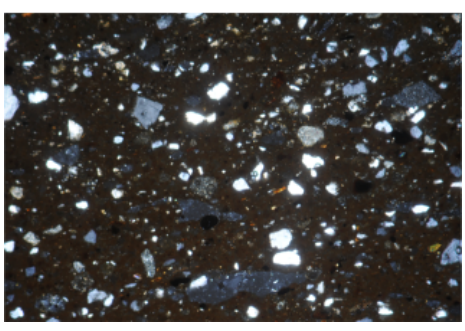

(a) Arcilla Marl

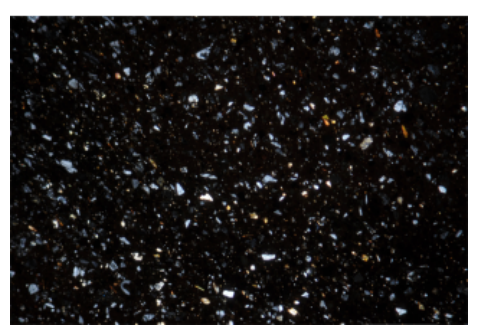

(b) Arcilla del Nilo

Fig. 3. Ejemplos de las imágenes de texturas de las piezas arqueológicas usadas en este trabajo.

Motor de búsqueda. Para proporcionar significancia estadística a los resultados, 33 experimentos independientes fueron realizados con cada configuración. El algoritmo genético (GA por sus siglas en inglés) fue usado como motor de búsqueda, y su forma básica es mostrada en la figura 4. Los parámetros de GA fueron fijados con base a nuestra experiencia y sus valores fueron los siguientes: tamaño de población: 50 individuos genotípicos, longitud del cromosoma: 50 codones generados aleatoriamente entre [0,255], número de generaciones: 200, método de selección: 2 padres con torneo binario, método de 
cruza: aleatorio a dos puntos, y método de mutación: mutación de un punto aleatorio de un sólo individuo por generación seleccionado aleatoriamente. En cada generación, la nueva población fue formada con 10 individuos seleccionados por elitismo, 15 nuevos individuos creados aleatoriamente y 25 generados por GA.

Tabla 1. Gramática BNF usada por GE.

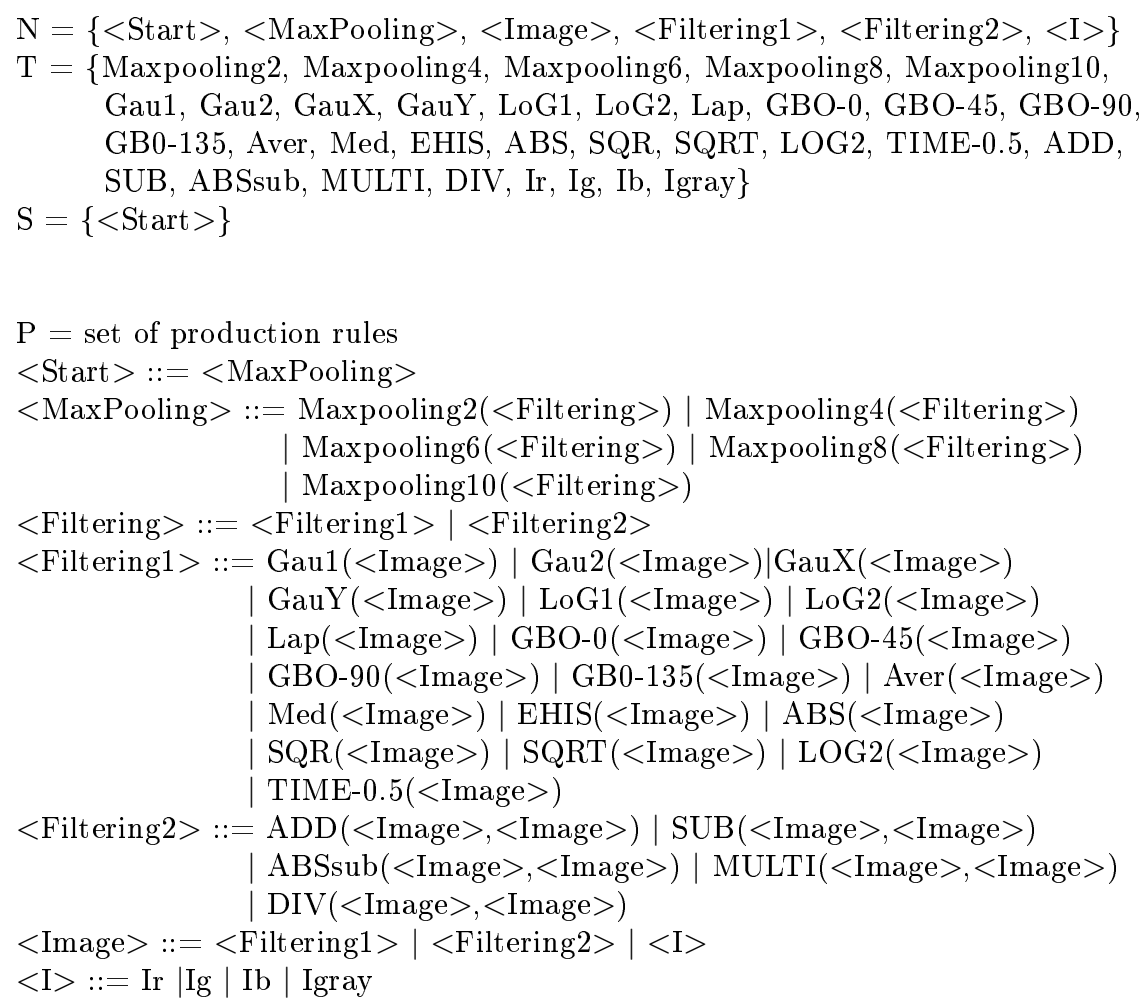

Salida. Es la representación de cada una de las imágenes de la base de datos y consiste en un vector de características obtenido mediante alguna de las dos representaciones utilizadas en este trabajo: histograma $(\mathrm{H})$ y estadísticos del histograma (EH), esté último formado por máxima probabilidad, energía, media, entropía, varianza, asimetría y $\mathrm{r}$ normalizada. Estas representaciones fueron obtenidas al aplicarse sobre las imágenes finales filtradas por los descriptores construidos por la GE. En total se obtuvieron 4 configuraciones, la gramática 
Una comparativa de diferentes parámetros en la construcción evolutiva de descriptores...

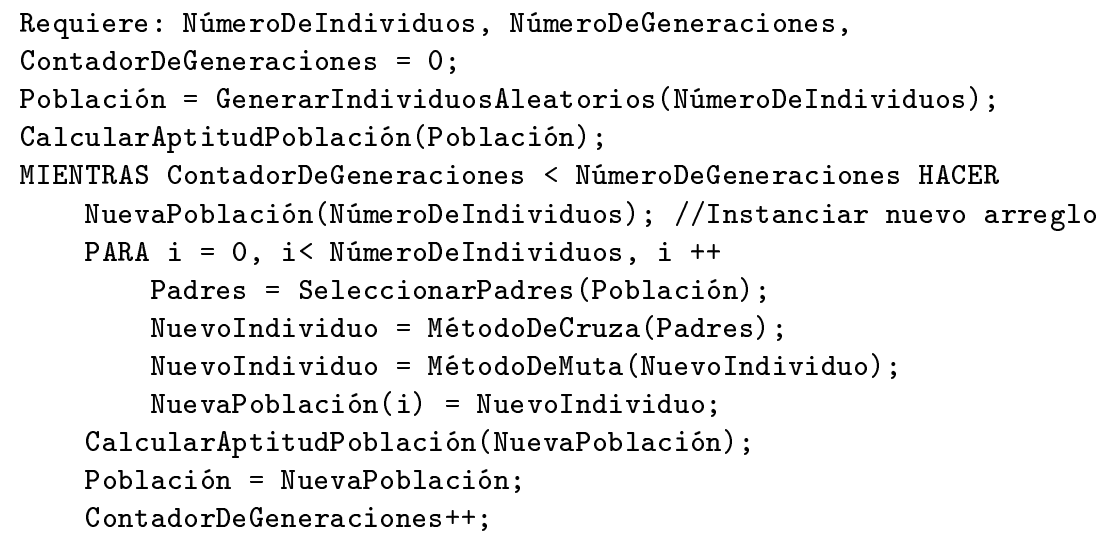

Fig. 4. Algoritmo Genético básico.

BNF1 con H y EH (BNF1-H y BNF1-EH respectivamente), y la gramática BNF2 con la aplicación de las mismas dos representaciones (BNF2-H y BNF2-EH).

Función objetivo. Para evaluar la aptitud de cada solución generada, la ecuación (2) [16] fue usada como función objetivo:

$$
\text { error }=1-\text { exactitud, }
$$

donde exactitud es el número de imágenes de prueba correctamente clasificadas dividido entre el total de imágenes de prueba. Se utilizó validación cruzada con 3 pliegues. Para cada uno de los 33 experimentos realizados con cada configuración, se hizo un muestreo aleatorio para formar los tres conjuntos de imágenes. La clasificación fue hecha por el algoritmo $\mathrm{kNN}$, con $\mathrm{k}=3$. Finalmente se promedió el error de clasificación de cada uno de los pliegues para obtener la aptitud de cada individuo evaluado. El fenotipo con el mínimo valor de la función objetivo corresponde a la mejor solución generada.

\section{Resultados}

La tabla 3 muestra la mediana y la varianza del porcentaje de clasificación de los 33 experimentos independientes para cada configuración, así como los porcentajes obtenidos por descriptores del estado del arte [15]. De los resultados obtenidos, se puede observar que en este experimento, los descriptores generados por GE obtuvieron un mejor desempeño.

En la tabla 4 se muestra el mejor descriptor generado por cada configuración y el porcentaje de clasificación obtenido.

La tabla 5 muestra el porcentaje del uso de cada canal de color, la escala de grises y la mezcla de éstos, lo cual fue calculado con los mejores descriptores 
Tabla 2. Descripción de los símbolos terminales usados por la gramática BNF.

\begin{tabular}{ll}
\hline Símbolos & \\
terminales & Descripción \\
\hline Maxpooling2 & Max-pooling 2D con una ventana de agrupado de tamaño 2X2 \\
Maxpooling4 & Max-pooling 2D con una ventana de agrupado de tamaño 4X4 \\
Maxpooling6 & Max-pooling 2D con una ventana de agrupado de tamaño 6X6 \\
Maxpooling8 & Max-pooling 2D con una ventana de agrupado de tamaño 8X8 \\
Maxpooling10 & Max-pooling 2D con una ventana de agrupado de tamaño 10X10 \\
Ir & Canal rojo de la imagen de entrada \\
Ig & Canal verde de la imagen de entrada \\
Ib & Canal azul de la imagen de entrada \\
Igray & Escala de grises de la imagen de entrada \\
Gau1 & Filtro de suavizado Gaussiano con una $\sigma=1$ \\
Gau2 & Filtro de suavizado Gaussiano con una $\sigma=2$ \\
GauX & Derivada a lo largo del eje X del filtro gaussiano \\
GauY & Derivada a lo largo del eje Y del filtro gaussiano \\
LoG1 & Laplaciano de filtro gaussiano con $\sigma=1$ \\
LoG2 & Laplaciano de filtro gaussiano con $\sigma=2$ \\
Lap & Filtro Laplaciano \\
GBO-0 & Filtro de Gabor con orientación de 0 grados \\
GBO-45 & Filtro de Gabor con orientación de 45 grados \\
GBO-90 & Filtro de Gabor con orientación de 90 grados \\
GB0-135 & Filtro de Gabor con orientación de 135 grados \\
Aver & Filtro de promediado con una ventana de agrupado de tamaño 5x5 \\
Med & Mediana en una ventana de agrupado de tamaño 5x5 \\
EHIS & Ecualización del histograma \\
ABS & Valor absoluto de una imagen píxel por píxel \\
ADD & Suma dos imágenes píxel por píxel \\
SUB & Resta dos imágenes píxel por píxel \\
ABSsub & Resta absoluta dos imágenes píxel por píxel \\
MULTI & Multiplicación de dos imágenes píxel por píxel \\
DIV & División de dos imágenes píxel por píxel pero regresa 0 sí el divisor es 0 \\
SQR & Cuadrado de una imagen píxel por píxel \\
SQRT & Raíz cuadrada de una imagen píxel por píxel \\
LOG2 & Logaritmo cuadrado de una imagen píxel por píxel \\
TIME-0.5 & Multiplicación de una imagen por 0.5 píxel por píxel \\
\hline &
\end{tabular}


Una comparativa de diferentes parámetros en la construcción evolutiva de descriptores...

Tabla 3. Comparación de los porcentajes de clasificación obtenidos por nuestra propuesta y por otros descriptores del estado del arte.

\begin{tabular}{|c|c|c|}
\hline \multirow[b]{2}{*}{ Configuraciones } & \multicolumn{2}{|c|}{ Datos } \\
\hline & Mediana & Varianza \\
\hline$\overline{B N F 1-H}$ & 0.8997 & 0.000091 \\
\hline$B N F 2-H$ & 0.8852 & 0.000253 \\
\hline$B N F 1-E H$ & 0.8681 & 0.000365 \\
\hline$B N F 2-E H$ & 0.8726 & 0.000181 \\
\hline Descriptores de Law [15] & 0.7391 & $*$ \\
\hline Filtros de Gabor [15] & 0.6812 & $*$ \\
\hline Descriptores de Haralick [15] & 0.7101 & $*$ \\
\hline
\end{tabular}

*El trabajo no reporta varianza

Tabla 4. Ejemplos de los mejores descriptores generados por las cuatro configuraciones de GE y sus respectivos desempeños.

\begin{tabular}{|c|c|c|}
\hline Configuración & Descriptor & $\begin{array}{l}\text { Porcentaje de } \\
\text { clasificación }\end{array}$ \\
\hline BNF1-H & $\begin{array}{l}\text { Maxpooling10(GBO- } \\
\text { 90(MULTI(Igray,DIV(LoG1(Igray),EHIS( } \\
\text { Igray)))) }\end{array}$ & $92.68 \%$ \\
\hline BNF2-H & $\begin{array}{l}\text { Maxpooling } 4 \text { (TIME- } \\
0.5(\text { MULTI(SUB(Igray,Igray),DIV(LoG1(GBO- } \\
135(\mathrm{DIV}(\mathrm{DIV}(\mathrm{SQR}(\mathrm{SQRT}(\mathrm{GBO}- \\
0(\text { Igray)})), \mathrm{DIV}(\mathrm{LOG} 2(\mathrm{ABS}(\text { Igray)}), \\
\text { Igray)),Igray))),SQR(Gau1(Igray)) )) ) }\end{array}$ & $92.68 \%$ \\
\hline BNF1-EH & $\begin{array}{l}\text { Maxpooling2(SQRT(MULTI(Igray,DIV(MULTI( } \\
\text { Ig,LoG2(ADD(Igray,SUB(SQRT(Ir),Igray)))), } \\
\text { Igray)))) }\end{array}$ & $90.22 \%$ \\
\hline BNF2-EH & $\begin{array}{l}\text { Maxpooling10(LoG1(LoG1(SUB(Igray,ADD( } \\
\text { MULTI(Igray,Igray),Igray))))) }\end{array}$ & $91.48 \%$ \\
\hline
\end{tabular}

generados en los 33 experimentos de las configuraciones de BNF1. De los resultados mostrados, se observa que BNF1-H utiliza más el canal verde mientras que BNF1-EH lo hace con la mezcla de canales.

Tabla 5. Porcentaje del uso de los canales de color, escala de gris y la mezcla de canales por las configuraciones de BNF1.

\begin{tabular}{lccccc}
\hline & & \multicolumn{4}{c}{ Canales de color } \\
Configuración & Rojo & Verde & Azul & Escala de grises & Mezcla de canales \\
\hline BNF1-H & $0 \%$ & $90.91 \%$ & $0 \%$ & $3.3 \%$ & $6.06 \%$ \\
BNF1-EH & $6.06 \%$ & $15.15 \%$ & $6.06 \%$ & $24.24 \%$ & $48.48 \%$ \\
\hline
\end{tabular}


Finalmente, la tabla 6 muestra la mediana, varianza, mínimo y máximo del número de símbolos terminales usados por los 33 mejores descriptores de cada configuración. Los resultados muestran que la configuración BNF1-H tiene una media de símbolos terminales usados menor que las otras configuraciones.

Tabla 6. Datos estadísticos del número de símbolos terminales usados por cada configuración.

\begin{tabular}{lcccc}
\hline & \multicolumn{4}{c}{ Datos estadísticos } \\
Configuración & Mediana & Varianza & Mínimo & Máximo \\
\hline BNF1-H & 3 & 12.309 & 3 & 18 \\
BNF2-H & 5 & 30.195 & 3 & 24 \\
BNF1-EH & 6 & 11.797 & 3 & 15 \\
BNF2-EH & 5 & 10.627 & 4 & 20 \\
\hline
\end{tabular}

\section{Conclusiones}

En el presente trabajo se mostró una metodología, basada en GE, la cual permite generar descriptores aplicados a la clasificación de imágenes de texturas de piezas arqueológicas.

En base a los resultados obtenidos, se puede afirmar que nuestra propuesta es viable de utilizar cuando no se tiene la suficiente información a priori de la base de datos del problema a tratar, pues para este experimento se obtuvieron desempeños mayores a los obtenidos por otros descriptores del estado del arte. Así también esta metodología puede ser usada en la solución de otros tipos de problemas de clasificación modificando el diseño de la gramática.

Con los datos que nos proporciona la tabla 3, podemos identificar que las configuraciones que utilizaron el histograma como parámetro de representación del vector de características, obtuvieron un mejor desempeño que las que utilizaron los estadísticos del histograma. También se observa que la configuración BNF1-H tiene una menor varianza que las demás configuraciones.

El presente trabajo busca servir de apoyo al arqueólogo para su toma de decisiones.

Como trabajo futuro, los autores proponen el diseño de gramáticas utilizando otros descriptores básicos, los cuales se pueden aplicar a diferentes bases de datos de imágenes de texturas. También se propone utilizar otros algoritmos de clasificación y motores de búsqueda en el proceso evolutivo.

Agradecimientos. Los autores agradecen el apoyo recibido del Tecnológico Nacional de México - Instituto Tecnológico de León y del Consejo Nacional de Ciencia y Tecnología (CONACyT) por medio de la beca para estudiantes de posgrado: 778561 (J. Jiménez) y CÁTEDRAS-2598 (A. Rojas). Así también se agradece a la Dra. Irmgard Hein el suministro de la base de datos de imágenes utilizada en este experimento. 
Una comparativa de diferentes parámetros en la construcción evolutiva de descriptores...

\section{Referencias}

1. Lu, D., Weng, Q.: A survey of image classification methods and techniques for improving classification performance. International Journal of Remote Sensing $28(5), 823-870$ (2007)

2. Mehta, R., Eguiazarian , K. E.: Texture Classification Using Dense Micro-Block Difference. IEEE Transactions on Image Processing 25(4), 1604-1616 (2016)

3. Sá Junior, J., Backes, A. R.: ELM based signature for texture classification. Pattern Recognition 51, 395-401 (2016)

4. Ledoux, A., Losson, O., Macaire, L.: Texture classification with fuzzy color co-occurrence matrices. In: 2015 IEEE International Conference on Image Processing (ICIP), pp. 1429-1433. IEEE, Quebec City (2015)

5. Zhang, Z., Liu, S., Mei, X., Xiao, B., Zheng, L.: Learning completed discriminative local features for texture classification. Pattern Recognition 67, 263-275 (2017)

6. Zhao, Y., Wang, R. G., Wang, W. M., Gao, W.: Local Quantization Code histogram for texture classification. Neurocomputing 207, 354-364 (2016)

7. Shao, L., Liu, L., Li, X.: Feature Learning for Image Classification Via Multiobjective Genetic Programming. IEEE Transactions on Neural Networks and Learning Systems 25(7), 1359-1371 (2014)

8. Tran, B., Zhang, M., Xue, B.: Multiple feature construction in classification on high-dimensional data using GP. In: 2016 IEEE Symposium Series on Computational Intelligence (SSCI), pp. 1-8. IEEE, Athens (2016)

9. Lensen, A., Al-Sahaf, H., Zhang, M., Xue, B.: Genetic Programming for Region Detection, Feature Extraction, Feature Construction and Classification in Image Data. Heywood, M., McDermott, J., Castelli, M., Costa, E., Sim, K. (eds) EuroGP 2016, LNCS, vol. 9594, pp. 51-67. Springer, Cham (2016).

10. Gavrilis, D., Tsoulos, I. G., Dermatas, E.: Selecting and constructing features using grammatical evolution. Pattern Recognition Letters 29(9), 1358-1365 (2008)

11. Ryan, C., Collins, JJ, O Neill, M.: Grammatical evolution: Evolving programs for an arbitrary language. In: Banzhaf, W., Poli, R., Schoenauer, M., Fogarty, T.C. (eds) EuroGP 1998, LNCS, vol. 1391, pp. 83-96. Springer, Heidelberg (1998)

12. Koza, J. R., Poli, R.: Genetic Programming. 1st edn. Springer US, Boston (2005)

13. Dempsey, I., O'Neill, M., Brabazon, A.: Foundations in Grammatical Evolution for Dynamic Environments. 1st edn. Springer Berlin Heidelber, Heidelber (2009)

14. Sotelo-Figueroa, M. A., Soberanes, H. J., Carpio, J. M., Fraire, H. J., Cruz, L., Soria-Alcaraz, J. A.: Improving the bin packing heuristic through grammatical evolution based on swarm intelligence. Mathematical Problems in Engineering 2014, 1-12, (2014)

15. Hein, I., Rojas-Domínguez, A., Ornelas, M., D'Ercole, G., Peloschek, L.: Automated classification of archaeological ceramic materials by means of texture measures. Journal of Archaeological Science: Reports 2017, Elsevier Press (2017)

16. Bradley, A. P.: The use of the area under the ROC curve in the evaluation of machine learning algorithms. Pattern Recognition 30(7), 1145-1159 (1997) 